\title{
The Study of Extended Extra Galactic Radio Sources: 2. The Study of Quasar 3c219 as a Nearest Extended Radio Source
}

\author{
Hovhannisyan MA1* $^{*}$, Andreasyan RR², Makhtesyan AP2, Paronyan \\ $\mathrm{GM}^{2}$, Abrahamyan $\mathrm{HV}^{2}$, Sargsyan $\mathrm{AV}^{1}$ and Aghadjanyan HS ${ }^{1}$ \\ ${ }^{1}$ NAS RA Institute of Applied Problems of Physics, Armenia \\ 2NAS RA V. Ambartsumian Byurakan Astrophysical Observatory (BAO), Armenia
}

\section{Research Article \\ Volume 3 Issue 4}

Received Date: November 18, 2019

Published Date: December 06, 2019

*Corresponding author: Hovhannisyan MA, NAS RA Institute of Applied Problems of Physics, Armenia, Email: martin@bao.sci.am

\section{Abstract}

The study of 3C 219 quasar and its surroundings was carried out. The distribution of galaxies, galaxies systems, and quasars inside the surface within a 360-minute radius is the same every- where. It turned out that the distribution in the 3C 219 is homogeneous.

Keywords: Extended Radio Sources; Quasars; Radio Galaxies; Homogeneous Distribution

\section{Introduction}

The quasar 3C219 is one of the 35 extended radio sources, of which 19 are quasars. It is the closest of those quasars. Its redshift is $\mathrm{z}=0.174732$ and it is a pretty bright optical source of $m=17,8 \mathrm{~m}$ magnitude. The study of quasar 3C219 is interesting because it has strong radiation in radio waves and large angular sizes, as it is far enough and has too large geometric dimensions [1]. To find out what features this quasar has it was done multi frequent investigations in all the areas studied so far. An extensive study was carried out using the all data of NASA database also (www.nasa.extragalactic database) [2]. It was studied not only the quasar, but also the extra galactic sources around it that are located within a radius of up to 360 minutes. In these regions were studied galactic pairs, triplets, groups, clusters, quasars, groups of quasars, gravity lenses, sources with absorption lines, sources with radiation lines, radio sources and supernovae stars, which could be obtained from NASA's sites [3]. The galaxies have been investigated for up to 60 minutes radius. For the regions with larger radius it is impossible to do, because the number of galaxies is too large. Within the 360-minute radius that number often exceeds one hundred thousand, and it is meaningless to involve so many galaxies in this study.

\section{Galaxies in the Environment of Quasar 3C219}

It is very important to find out the density distribution of galaxies 'in the environment of quasar. We've scanned the regions from 1 minute to 60 minutes. Table 1 presents data in the following sequence: The first column is the distance from the quasar center in minutes; in the second, the number of galaxies inside that circle; in the third column the ratio of the surface to the surface of the region with a radius of one minute; the fourth is the number of galaxies inside the ring surrounded by neighboring radii, in the fifth the galaxy density in the ring, in the sixth column the galactic density within the corresponding radius. As seen from the table, the density of galaxies decreases with the distance from quasar. The comparison of five and six column allows us to understand that the 


\section{Physical Science \& Biophysics Journal}

density of galaxies in the quasar environment is large. This is possible if the presence of the main part of galaxies in the quasar environment is conditioned by the interaction with quasar. If the galaxies were distributed uniform lee in the direct environment of quasar, the number of galaxies would be not more greater. Since not for all galaxies we have the redshifts, it is not possible to speak about the immediate environment of the quasar, so we discussed in terms of single spherical distribution, especially when the relative number of galaxies with known red shifts is small.

\begin{tabular}{|c|c|c|c|c|c|}
\hline $\mathbf{R}$ & $\mathbf{N}$ & $\mathbf{S}$ & $\mathbf{N 1}$ & $\mathbf{N 1 / S}$ & $\mathbf{N} / \boldsymbol{\pi R} \mathbf{2}$ \\
\hline 1 & 22 & 1 & 22 & 22 & 15 \\
\hline 2 & 60 & 3 & 38 & 12.7 & 11.6 \\
\hline 3 & 104 & 5 & 44 & 8.8 & 11.6 \\
\hline 4 & 186 & 7 & 82 & 11.7 & 10.8 \\
\hline 5 & 270 & 9 & 84 & 9.3 & 10.4 \\
\hline 6 & 373 & 11 & 103 & 9.4 & 9.8 \\
\hline 7 & 474 & 13 & 101 & 9.2 & 9.6 \\
\hline 8 & 613 & 15 & 139 & 9.3 & 9.4 \\
\hline 9 & 755 & 17 & 142 & 8.4 & 9.3 \\
\hline 10 & 930 & 19 & 175 & 9.2 & 8.5 \\
\hline 20 & 3415 & 300 & 2485 & 8.3 & 8.3 \\
\hline 30 & 7432 & 500 & 4017 & 8 & 8.8 \\
\hline 40 & 14144 & 700 & 6712 & 9.6 & 8.1 \\
\hline 50 & 20260 & 900 & 6116 & 6.8 & 8 \\
\hline 60 & 28901 & 1100 & 8641 & 7.9 & \\
\hline
\end{tabular}

Table 1: Galaxies in the environment of quasar 3C219 till the radius of 60 minutes.

In Table 2 we bring the number of different objects in the regions with different radius $\mathrm{R}$. The designations in the first line are: GP for galactic pairs, GT - galactic triplets, GG - galactic groups, GC - galactic cluster, Q quasars, QG \{ groups of groups, GL - gravity lens, AbLS - sources with absorption lines, EmLS - sources with radiation lines. In the first column there are the radius of regions in minutes, and the number of corresponding objects in the other columns.

\begin{tabular}{|c|c|c|c|c|c|c|c|c|}
\hline $\mathbf{R}$ & GP & GT & GG & GC & QG & GL & AbLS & EmLS \\
\hline 60 & 2 & 2 & 28 & 93 & 0 & 0 & 0 & 0 \\
\hline 120 & 4 & 10 & 92 & 318 & 0 & 2 & 2 & 0 \\
\hline 180 & 9 & 19 & 224 & 612 & 0 & 5 & 5 & 0 \\
\hline 240 & 17 & 34 & 357 & 1050 & 0 & 6 & 8 & 3 \\
\hline 300 & 27 & 63 & 608 & 1662 & 0 & 6 & 19 & 16 \\
\hline 360 & 44 & 93 & 904 & 2411 & 0 & 10 & 27 & 35 \\
\hline
\end{tabular}

Table 2: The number of systems of galaxies in the environment of quasar 3C219.

Table 2a shows the concentrations of these same objects. Table 3 shows the number of radio sources in the environment of the quasar, their concentrations inside the corresponding radius and within the ring between the neighboring radius. It turns out that the density of radio sources decreases with the distance from the quasar. This can be due to the fact that there is accumulation of radio sources inside in the neighborhood of quasar 3C219, 


\section{Physical Science \& Biophysics Journal}

which was expected. In Table 4, the number and concentrations of other quasars are given in the environment of quasar 3C219. Apparently, the distribution of quasars is not subject to any regularity; these quasars are independent of each other and are independent of the 3C219 quasar.

\begin{tabular}{|c|c|c|c|c|c|c|c|c|}
\hline $\mathbf{R}$ & $\mathbf{G P ~ 1 0}^{-4}$ & $\mathbf{G T ~ 1 0}^{-4}$ & $\mathbf{G G ~ 1 0}^{-3}$ & $\mathbf{G C ~ 1 0}^{-2}$ & $\mathbf{Q G ~ 1 0}$ & $\mathbf{G L ~ 1 0}^{-4}$ & AbLS 10-4 $^{-4}$ & EmLS 10-4 $^{-4}$ \\
\hline 60 & 5.6 & 5.6 & 7.8 & 2.6 & 0 & 0 & 0 & 0 \\
\hline 120 & 2.8 & 6.9 & 6.4 & 2.2 & 0 & 1.4 & 1.4 & 0 \\
\hline 180 & 3 & 5.9 & 6.9 & 1.9 & 0 & 1.5 & 1.5 & 0 \\
\hline 240 & 3 & 5.9 & 6.2 & 1.8 & 0 & 1 & 1.4 & 0.5 \\
\hline 300 & 3 & 7 & 6.8 & 1.8 & 0 & 0.7 & 2.1 & 1.8 \\
\hline 360 & 3.4 & 7.2 & 7 & 1.9 & 0 & 0.8 & 2.1 & 2.7 \\
\hline
\end{tabular}

Table 2a: The density of systems of galaxies is in the environment of the quasar 3C219.

\begin{tabular}{|c|c|c|c|c|}
\hline 1 & 3 & 1 & 3 & 3 \\
\hline 2 & 8 & 3 & 0.9 & 1.7 \\
\hline 3 & 8 & 5 & 0.5 & 0 \\
\hline 4 & 8 & 7 & 0.4 & 0.11 \\
\hline 5 & 9 & 9 & 0.53 & 0.36 \\
\hline 6 & 13 & 11 & 0.35 & 0.31 \\
\hline 7 & 17 & 13 & 0.28 & 0.07 \\
\hline 8 & 18 & 15 & 0.26 & 0.18 \\
\hline 9 & 21 & 17 & 0.25 & 0.21 \\
\hline 10 & 25 & 19 & 0.13 & 0.09 \\
\hline 20 & 53 & 300 & 0.08 & 0.04 \\
\hline 30 & 74 & 700 & 0.08 & 0.07 \\
\hline 40 & 122 & 900 & 0.07 & 0.06 \\
\hline 50 & 174 & 1100 & 0.07 & 0.07 \\
\hline 60 & 252 & 10800 & 0.06 & 0.05 \\
\hline 120 & 835 & 18000 & 0.12 & 0.05 \\
\hline 180 & 1720 & 25200 & 0.06 & 0.06 \\
\hline 240 & 3216 & 32400 & 0.06 & 0.07 \\
\hline 300 & 5477 & 39600 & 0.07 & 0.07 \\
\hline 360 & 8435 & & & \\
\hline
\end{tabular}

Table 3: Radio sources in the environment of quasar 3C219.

The number of quasars depends on the distance to such extent as it is possible to detect quasars at these distances. So the number of other quasars around the quasar 3C219 does not decrease, but diminishes the likelihood of detecting them. In the environment of quasar 3C219 there are other quasars at all possible distances. We can say that in this region the space is homogeneous.
Of course there are regions where it is not so. About this a separate article will be presented.

In Figure 1 we bring all the known, full observational data for the quasar 3C219. From the spectra of the quasar $3 \mathrm{C} 219$ it is clear that the very definite ratio $\lg \left(\mathrm{f}_{v}\right) / \lg (v)$ is maintained by the whole region of electromagnetic waves [4-10]. 


\section{Physical Science \& Biophysics Journal}

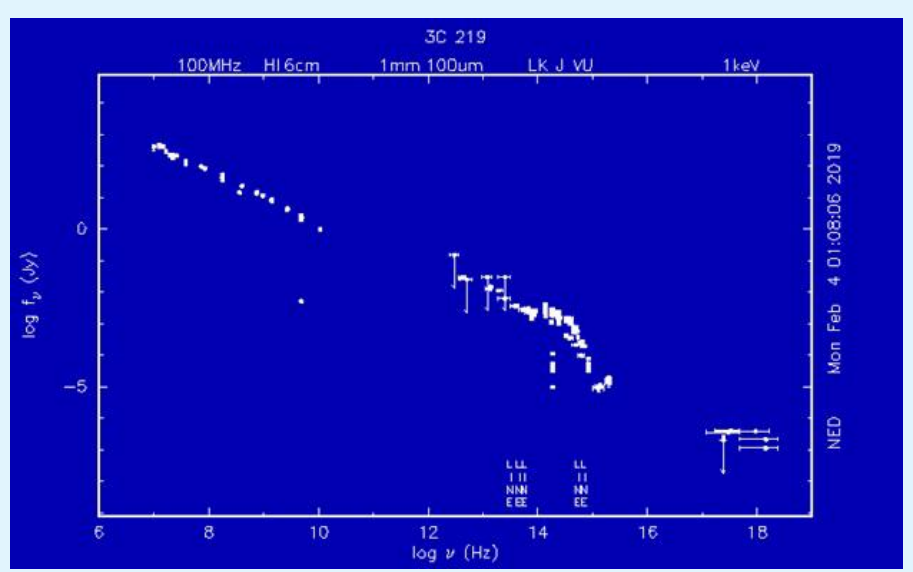

Figure 1: The intensity of the quasar 3C 219 in the frequencies from $10 \mathrm{MHz}$ to $10 \mathrm{KEV}$.

\section{Conclusion}

The distribution of extra galactic sources in the conical volume in a 360-minute radius and up to redshift $\mathrm{z}=6$ can be considered as homogeneous. Consequently, the quasar 3C219 is located in such a region where other cosmic objects also have the same characteristics as the quasar 3C219.

Figure 2 shows the distribution of quasars by their red shifts. The picture shows the existence of two maxima where there is a certain deficiency of the number of quasars in the regions of $\mathrm{z} \sim 0.7-1.7$. In the case of red shifts with greater value, the deficit of quasars is conditioned by the deficit of opportunity to find relatively weak quasars, and in the case of smaller ones there are no quasars. Naturally, that the deficit of quasars in closest space is conditioned by the absence of quasars in those regions. The image of the 3C219 quasar environment is very in line with the perceptions of the homogeneous universe, with only one deviation that at some distances there is a weakly deficient. We have such an image at the NGC 6251 radio galaxy [11]. In this domain, the quasidistribution divides between the relative distributions, in contrast to the four distinct domains, where there are large-scale inequalities different from the homogeneous cosmos. In the region near to NGC 6251 the distribution of quasars is near to homogeneous, in difference to the four distinct domains where there are large scale inhomogeneous.

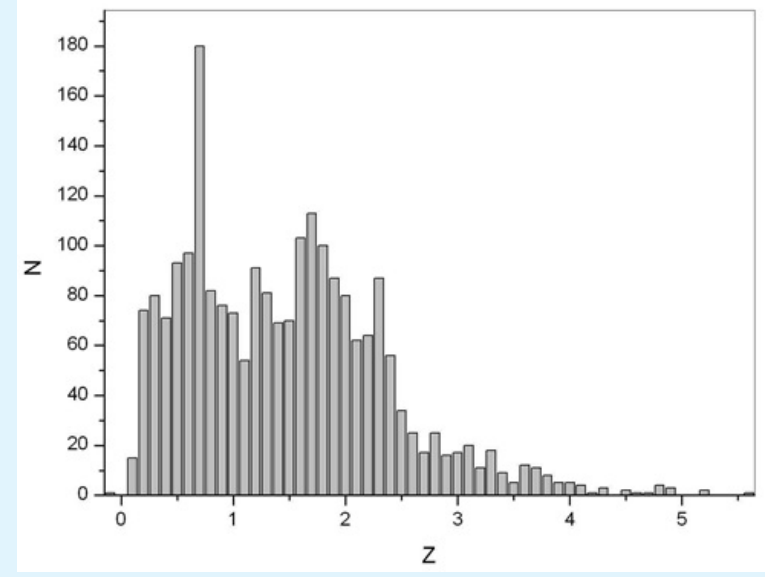

Figure 2: The distribution of quasars by their red shifts. 


\section{Physical Science \& Biophysics Journal}

\begin{tabular}{|c|c|c|c|c|}
\hline 1 & 1 & 1 & 1 & 1 \\
\hline 2 & 2 & 3 & 0.5 & 0.33 \\
\hline 3 & 2 & 5 & 0.22 & 0 \\
\hline 4 & 2 & 7 & 0.13 & 0 \\
\hline 5 & 2 & 9 & 0.08 & 0 \\
\hline 6 & 3 & 11 & 0.08 & 0.09 \\
\hline 7 & 3 & 13 & 0.06 & 0 \\
\hline 8 & 3 & 15 & 0.05 & 0 \\
\hline 9 & 3 & 17 & 0.04 & 0 \\
\hline 10 & 5 & 19 & 0.05 & 0.11 \\
\hline 20 & 10 & 300 & 0.03 & 0.02 \\
\hline 30 & 23 & 500 & 0.03 & 0.03 \\
\hline 40 & 38 & 700 & 0.02 & 0.02 \\
\hline 50 & 55 & 900 & 0.02 & 0.02 \\
\hline 60 & 72 & 1100 & 0.02 & 0.02 \\
\hline 120 & 222 & 10800 & 0.02 & 0.01 \\
\hline 180 & 486 & 18000 & 0.02 & 0.01 \\
\hline 240 & 886 & 25200 & 0.02 & 0.02 \\
\hline 300 & 1426 & 32400 & 0.02 & 0.02 \\
\hline 360 & 2123 & 39600 & 0.02 & 0.02 \\
\hline
\end{tabular}

Table 4: Other quasars in the environment of quasar 3C219.

\section{References}

1. Burch SF (1979) Multifrequency studies of seven 3CR radio sources-II. Results and interpretation. Monthly Notices of the Royal Astronomical Society 186(3): 519-553.

2. NASA Extragalactic Database.

3. NASA/IPAC Extragalactic Database (NED), Jet Propulsion Laboratory, California Institute of Technology.
4. Artyukh VS, Ogannisyan MA, Tyul'bashev SA (1994) AstL 20: 146.

5. Kassim NE, Joseph T, Lazio W, Erickson WC, Perley RA, et al. (2007) The $74 \mathrm{MHz}$ System on the Very Large Array. The Astrophysical Journal Supplement Series 172(2): 686.

6. Nath BB (2010) Extended X-ray emission from radio galaxy cocoons. Monthly Notices of the Royal Astronomical Society 407(3): 1998-2006.

7. Toba Y, Oyabu S, Matsuhara H, Malkan MA, Gandhi P, et al. (2014) Luminosity And Redshift Dependence Of The Covering Factor Of Active Galactic Nuclei Viewed With Wise And Sloan Digital Sky Survey. The Astrophysical Journal 788(1): 45.

8. Minjin K, Ho LC, Peng CY (2017) ApJS 32: 21.

9. Baldi RD, Rodriguez ZJ, Chiaberge M, Capetti A, Sparks WB, et al. (2019) Hubble Space Telescope Emissionline Images of nearby 3CR Radio Galaxies: Two Photoionization, Accretion, and Feedback Modes. The Astrophysical Journal 870(1): 53.

10. Lyu J, Rieke GH (2018) Polar Dust, Nuclear Obscuration, and IR SED Diversity in Type-1 AGNs. The Astrophysical Journal 866(2): 92.

11. Hovhannisyan MA, Andreasyan RR, Paronyan GM (2018) Investigations of the extended radio galaxy NGC 6251 and the galaxies in the environment. Communications of BAO 2(2): 398-400. 